\title{
Sensitivity of Candida albicans from patients with chronic oral candidiasis
}

\author{
W.P. HoLBROOK* \\ R. KIPPAX \\ Department of Oral Medicine, Turner Dental School, Bridgeford Street, Manchester M15 6FH
}

\begin{abstract}
Summary
Many patients with oral candidiasis respond very slowly or not at all to therapy with amphotericin. Strains of Candida albicans were collected from 17 patients clinically resistant and from 15 who responded to a normal course of amphotericin treatment. Minimal inhibitory concentrations (MIC) determined on diagnostic sensitivity test agar plates gave values of: amphotericin $0.5 \mathrm{mg} / \mathrm{l}$; nystatin 50 i.u./ml; chlorhexidine $12.5 \mathrm{mg} / \mathrm{l}$. No clear MIC could be determined with plates containing miconazole. No difference was noted in MIC values between the 2 groups of patients. Tube-dilution tests in Sabouraud's broth gave MIC values of : amphotericin $0.25 \mathrm{mg} / \mathrm{l}$; nystatin 12.5 i.u./ml; chlorhexidine $1.5 \mathrm{mg} / \mathrm{l}$; miconazole 8-32 $\mathrm{mg} / \mathrm{l}$; ketonazole $64 \mathrm{mg} / \mathrm{l}$. Persistence of oral candidiasis is not an indication of infection with resistant organisms. Despite difficulties in in vitro sensitivity testing of miconazole a clinical trial of the drug for treating oral candidiasis is indicated.
\end{abstract}

\section{Introduction}

Chronic candidiasis may present in the mouth as: denture stomatitis and angular cheilitis, which can be termed chronic atrophic candidiasis; chronic hyperplastic candidiasis, sometimes termed candida leucoplakia; oral manifestations of chronic mucocutaneous candidiasis, sometimes associated with endocrinopathy.

In the Oral Medicine Clinic in Manchester Dental School, denture stomatitis and chronic hyperplastic candidiasis were most frequently encountered. Denture stomatitis is seen as an erythematous area of the palate, with a shape directly related to the extent of the denture-bearing area. The mucosa is atrophic and candidal pseudomembrane is not seen. Swabs and smears taken from the palate would be strongly positive for Candida. Chronic hyperplastic candidiasis appears as a raised adherent white patch on the mucosa which is sometimes regarded as a pre-malignant lesion. Candida is thought to be the aetiological agent and can be detected on culture and in biopsy material stained with PAS reagent.

Clinical experience suggested that many patients with these 2 conditions responded slowly or not at

\footnotetext{
*Present address: Central Microbiological Laboratories, Western General Hospital, Crewe Road, Edinburgh EH4 2XU
}

all to therapy with amphotericin lozenges, $10 \mathrm{mg} 4$ times daily, which was often replaced by or supplemented with nystatin or amphotericin cream. Many patients received these drugs for more than 2 months and several had been treated for more than one year.

Miconazole has been shown by Brincker (1977) to be a useful drug in the treatment of oral candidiasis in debilitated patients and it was decided to include this drug and a miconazole analogue, ketoconazole, in an in vitro study of the sensitivity of oral clinical isolates of $C$. albicans to a variety of anti-candidal agents.

Materials and methods

Strains

Thirty-two strains of $C$. albicans were collected $\mathbb{D}$ 17 from patients who had been treated with ampho $\frac{\mathbb{Q}}{\mathbb{D}}$ tericin for more than 2 months without clinical ov 3 mycological cure, and 15 strains from new patients who responded to a normal course of treatment lasting approx. 2 weeks.

\section{Sensitivity tests}

The anti-candidal drugs used included amphotericin, miconazole, ketoconazole, nystatin and chlorhexidine, which was shown by Langslet et al. (1974) to be an effective anti-candidal preparation in a clinical trial of debilitated children with oral candidiasis.

\section{Tests on solid medium}

Sterile amphotericin for injection was dissolved and diluted in sterile saline. Sterile nystatin and miconazole powders were dissolved in dimethyl sulphoxide, and $0.2 \%$ chlorhexidine gluconate liquid was diluted in sterile distilled water; ketoconazole was not tested on solid medium. Appropriate dilutions of each agent were added to batches of molten diagnostic sensitivity test (DST) agar (Oxoid) to give doubling dilutions of the agents when dispensed in 20-ml into Petri dishes. Control plates containing solvent alone were also prepared.

One drop $(c .0 .02 \mathrm{ml})$ of each of the test strains was used to inoculate 10-ml of Sabouraud's broth and incubated for $5 \mathrm{hr}$ at $37^{\circ} \mathrm{C}$. One drop of this culture was used to inoculate the agar plates. Counts were made of the test cultures and were approximately $1 \times 10^{7}$ organisms $/ \mathrm{ml}$ giving an inoculum 
size of approximately $2 \times 10^{5}$ organisms. All plates were incubated overnight at $37^{\circ} \mathrm{C}$ and examined for growth. The lowest concentration of agent in the plates on which no growth was detected was recorded as the MIC value.

\section{Tests in liquid medium}

Sensitivity testing was also performed in liquid medium. Eighteen further isolates of $C$. albicans, 7 from clinically resistant patients and 11 from patients who responded to a normal course of treatment, were used to determine the MIC of amphotericin, nystatin and chlorhexidine. All 50 isolates were used in broth dilution tests with miconazole and ketoconazole.

The agents were dissolved and diluted as described for the tests on solid medium, ketoconazole was treated as for miconazole. Doubling dilutions of the agents were prepared in Sabouraud broth, and control tubes containing broth and solvent alone were also set up. Test cultures were prepared as previously described and one drop $(c .0 .02 \mathrm{ml})$ used to inoculate each tube. The inoculum size gave a concentration of approximately $2 \times 10^{5}$ organisms $/ \mathrm{ml}$ in each tube. Incubation was overnight at $37^{\circ} \mathrm{C}$. The MIC was determined as the lowest concentration of agent at which no growth of $C$. albicans was visible. Minimal Candida-cidal (MCC) values were determined as the lowest concentration of agent from which no growth was noted on subculture of the test culture on to Sabouraud's agar.

\section{Results}

The MIC values of 32 strains of $C$. albicans obtained on DST agar media are: amphotericin $0.5 \mathrm{mg} / \mathrm{l}$; nystatin 50 i.u. $/ \mathrm{ml}$; chlorhexidine $12.5 \mathrm{mg} / \mathrm{l}$ and miconazole $>250 \mathrm{mg} / \mathrm{l}$. No difference in results was noted between the strains isolated from patients clinically resistant to traditional therapy and those

TABLE 1. MIC and MCC (minimal Candida-cidal) values of 18 strains of C. albicans tested in Sabouraud's broth

\begin{tabular}{llcc}
\hline & & MIC & MCC \\
\hline Amphotericin & $\mathrm{mg} / \mathrm{l}$ & $0 \cdot 25$ & 1 \\
Nystatin & i.u./ml & $12 \cdot 5$ & $12 \cdot 5$ \\
Chlorhexidine & $\mathrm{mg} / \mathrm{l}$ & 1.5 & 3 \\
\hline
\end{tabular}

who responded to a normal course of amphotericin or nystatin. Problems were encountered with the miconazole test and no inhibition of the test strains of $C$. albicans was noted in concentrations up to 250 $\mathrm{mg} / \mathrm{l}$ of the drug at which level miconazole began to precipitate out in the agar medium. Because of these problems no test was carried out on solid media with the miconazole analogue ketoconazole.

Values for MIC and MCC obtained with the 18 additional test strains in liquid medium are given in Table 1. No difference in MIC and MCC values was noted between strains from patients clinically resistant or clinically sensitive to amphotericin and nystatin. There was a reduction in the MIC values in Sabouraud's broth compared with those on DST agar. This was a matter of one dilution, i.e. within the limits of error of the system with amphotericin but was 2 dilutions with nystatin and considerably greater with chlorhexidine.

A range of MIC and MCC values was obtained with miconazole and ketoconazole tested with all 50 strains (Tables 2 and 3). This did not, however, reflect the clinical sensitivity or resistance of the organism to the other anti-candidal drugs. The MCC values were approximately one dilution higher than the MIC values. Ketoconazole gave higher MIC and MCC values than did miconazole.

TABLE 2. Range of MIC and MCC (minimal Candida-cidal) values obtained with 50 strains of $C$. albicans tested in broth medium containing miconazole

\begin{tabular}{ccc}
\hline \multirow{2}{*}{$\begin{array}{c}\text { Miconazole } \\
(\mathrm{mg} / \mathrm{l})\end{array}$} & \multicolumn{2}{c}{ No. of strains } \\
\cline { 2 - 3 } & MIC & MCC \\
\hline 8 & 13 & 6 \\
16 & 17 & 13 \\
32 & 20 & 29 \\
64 & 0 & 2 \\
\hline
\end{tabular}

TABLE 3. Range of MIC and MCC (minimal Candida-cidal) values obtained with 50 strains of $C$. albicans tested in broth medium containing ketoconazole

\begin{tabular}{ccc}
\hline \multirow{2}{*}{$\begin{array}{c}\text { Ketoconazole } \\
(\mathrm{mg} / \mathrm{l})\end{array}$} & \multicolumn{2}{c}{ No. of strains } \\
\cline { 2 - 3 } & MIC & MCC \\
\hline 32 & 18 & 3 \\
64 & 30 & 13 \\
128 & 2 & 32 \\
$>128$ & 0 & 2 \\
\hline
\end{tabular}

\section{Discussion}

True resistance of clinical isolates of $C$. albicans to the traditional anti-candidal drugs amphotericin and nystatin is not recognized and it was unlikely that these isolates would be any different in that respect. It is well known, however, that candidal infections can be difficult to eradicate. Host factors play a significant part in the course of any infection with $C$. albicans. Several of the patients in this study were elderly, a few were diabetic or on steroid therapy, but many had no known predisposing factor to indicate the cause of such prolonged candidiasis. 
Mackie, Parratt and Jenkins (1978) have studied 8 patients with resistant oral candidiasis and found 4 to have defects of specific Candida-related immune function but no generally detectable abnormality of their immune system. Three of these patients with chronic hyperplastic candidiasis were successfully treated with surgical excision of the lesions. The other 4 patients were found to have no Candidarelated immune defect but they were deficient in either folate or iron. Replacement therapy and long-term treatment with anti-candidal drugs was given. In the present study, all patients received a routine simple haematological screen and many had tests for folate, vitamin $B_{12}$ and iron levels. Any patients in whom abnormal results were found were referred to a physician for replacement therapy. Resolution of the candidiasis occurred in some but not all of these patients.

The MIC values obtained for the 50 strains tested with miconazole and ketaconazole are higher than may be expected from most reports in the literature. In a review Milne (1978) noted the accepted MIC value for miconazole to be $2 \mathrm{mg} / \mathrm{l}$. MacFarlane, Ferguson and Mackenzie (1978) reported MIC values of $0.5 \mathrm{mg} / 1$ in their study of $C$. albicans isolated from patients with angular cheilitis. Their tests were carried out with solutions of miconazole in 'fish-spines' placed on nutrient agar pour-plates. These authors stated that higher MIC values were obtained when the organism was grown on the surface of agar plates containing miconazole.

Shadomy et al. (1977) determined MIC values of miconazole with clinical and laboratory isolates of C. albicans on Sabouraud's agar. They found that whilst several laboratory strains were sensitive to one $\mathrm{mg} / \mathrm{l}$ of miconazole, most clinical isolates were resistant to a $16 \mathrm{mg} / \mathrm{ml}$ concentration. This latter figure is in agreement with the results of the present study of clinical isolates. Van Cutsem and Thienpont (1972) reported MIC values of $1-10 \mathrm{mg} / \mathrm{l}$ and Holt and Azimi (1978) reported one clinical isolate found to be resistant to miconazole with a MIC value of $100 \mathrm{mg} / \mathrm{l}$. A feature of several reports of MIC values of miconazole is the fairly broad range of results within any one group of strains tested by one particular method; in the present study a more gradual inhibition of growth with increasing miconazole concentration contrasted sharply with the clearly defined MIC values obtained with the other agents tested. The discrepancies between reported MIC values support the conclusion of Shadomy et al. (1977) that there is considerable difficulty in achieving reproducibility of results with miconazole in different test procedures. This discrepancy is not so marked with amphotericin or nystatin as the results of broth and agar-dilution methods reported here indicate. Some lack of reproducibility between test methods was, however, evident with chlorhexidine and although several subsequent reports have supported the view of Langslet et al. (1974) that chlorhexidine is effective in the treatment of oral candidiasis, this does not agree with the findings of Sharon, Berdicevsky and Ben-Aryeh (1977).

Efforts to try to improve the reproducibility and clarity of the MIC test for miconazole have indicated, at least provisionally, that variations in the media, agar or broth dilution methods, and the $\mathrm{pH}$ of the medium all greatly affect the MIC values obtained. Shadomy et al. (1977) have also shown that inoculum size is especially critical in determining the MIC of miconazole with C. albicans.

Despite the difficulties in establishing a reliable test of sensitivity to miconazole this drug has been shown by Brincker (1977) to be a useful agent in the treatment of oral candidiasis in debilitated patients who are typically resistant to traditional therapy. Further clinical trials are indicated for debilitated patients and those with stubborn chronic hyperplastic candidiasis. The studies of Mackie et al. (1978) would help identify those patients likely too respond to nutritional replacement and long-term? anti-candidal drug therapy and also those patients from whom surgical excision of the lesions was likely to be the only successful treatment.

\section{Acknowledgments}

The financial assistance of Janssen Pharmaceutical Ltd Marlow, Bucks, is gratefully acknowledged.

\section{References}

BrINCKer, H. (1977) Miconazole in oral candidiasis Proceedings of the Royal Society of Medicine, 70 (Suppl. 1), 29.

Holt, R.J. \& AzImI, A. (1978) Miconazole-resistant Candida. (Correspondence.) Lancet, i, 50.

Langslet, A., Olsen, I., Lie, S.O. \& LøKken, P. (1974) Chlorhexidine treatment of oral candidiasis in seriously diseased children. Acta paediatrica scandinavica, 63, 809.

MacFarlane, T.W., Ferguson, M.M. \& Mackenzie, D. (1978) Sensitivity to miconazole of microorganisms associated with angular cheilitis. British Dental Journal, 144, 199.

Mackie, R.M., Parratt, D. \& Jenkins, W.M.M. (1978) The relationship between immunological parameters and response to therapy in resistant oral candidosis. British Journal of Dermatology, 98, 343.

MiLNE, L.J.R. The antifungal imidazoles: clotrimazole and miconazole. Scottish Medical Journal, 23, 149.

Shadomy, S., Paxton, L., Espinel-Ingroff, A. \& Shadomy, H.J. (1977) In vitro studies with miconazole and miconazole nitrate. Journal of Antimicrobial Chemotherapy, 3, 147.

Sharon, A., Berdicevsky, I. \& Ben-Aryeh, H. (1977) The effect of chlorhexidine mouth rinses on oral Candida in a group of leukemic patients. Oral Surgery, Oral Medicine and Oral Pathology, 44, 201.

Van Cutsem, M.M. \& ThienPont, D. (1972) Miconazole, a broad-spectrum antimycotic agent with antibacterial activity. Chemotherapy, 67, 392. 